\section{IDDF2018-ABS-0117 IDENTIFICATION OF MOLECULES DRIVING HEPATIC METASTASIS FORMATION OF GASTRIC CANCER CELLS}

Mitsuro Kanda*, Chie Tanaka, Daisuke Kobayashi, Masaya Suenaga, Hideki Takami, Norifumi Hattori, Yukiko Niwa, Masamichi Hayashi, Suguru Yamada, Goro Nakayama, Masahiko Koike, Michitaka Fujiwara, Yasuhiro Kodera. Department of Gastroenterological Surgery (Surgery II), Nagoya University Graduate School of Medicine, Japan

\subsection{6/gutjnl-2018-IDDFabstracts.12}

Background Hepatic metastasis and relapse contribute to the high incidence of gastric cancer (GC)-related fatalities and represent a frequent and crucial problem for oncologists. The aim of this study was to identify and explore diagnostic biomarkers and molecular targets to predict and treat hepatic metastases.

Methods Transcriptome and bioinformatics analyses were conducted using tissues from patients who had GC with synchronous hepatic metastasis to identify a candidate molecule that specifically mediates hepatic metastasis of GC. Stable knockout cells were established by genome editing using the CRISPRCAS9 system. In vitro analyses including proliferation, apoptosis, migration, invasion and adhesion assays were performed to evaluate the effect of knockout. Mouse subcutaneous xenograft and liver metastasis models were used to evaluate influences of knockout on tumorigenicity and formation of hepatic metastasis. mRNA levels of the candidate molecule were determined in the gastric tissues of 200 patients with GC to assess whether differential gene expression predicted patient prognosis.

Results Synaptotagmin VII (SYT7) was identified as a candidate biomarker for hepatic metastasis in GC. GC cell lines exhibited differential SYT7 expression levels and the mRNAs encoding SNAI1 and TGFB3 was expressed at levels that correlated significantly with those of SYT7 mRNA, whereas the expression levels of RGS2 mRNA correlated inversely with those of SYT7. The proliferation, invasion, adhesion and migration abilities of stable SYT7-knockout GC cells were significantly reduced compared to MKN control cells. Stable SYT7-knockout GC cells showed significantly more annexin V positive cells in comparison to the MKN1 cells. Tumorigenicity of GC cells in mouse subcutaneous xenograft models was significantly decreased in stable SYT7-knockout GC cells accompanying reduced $\mathrm{Bcl}-2$ and HIF-1a protein expression in subcutaneous tumours. Tumour growth after portal injection of cells was significantly decreased in stable SYT7-knockout GC cells. High SYT7 expression in clinical GC tissues was associated with shortened overall and disease-free survival.

Conclusions SYT7 represents a promising target for treatment of hepatic metastasis of GC.

\section{IDDF2018-ABS-0121 INNOVATIVE MASS SPECTROMETRY PROBE VIA POLARITY-REVERSAL DERIVATIZATION FOR MAPPING GLOBAL CARBOXYL- CONTAINING METABOLITES IN COLORECTAL CANCER HUMAN SERUM}

Jian-lin Wu*, Xiqing Bian, Na Li, Wen Luan Wendy Hsiao. State Key Laboratory of Quality Research in Chinese Medicine, Macau University of Science and Technology, Macau

\subsection{6/gutjnl-2018-IDDFabstracts. 13}

Background Carboxyl-containing metabolites (CCMs) widely exist in the living system and are the essential components of life. Global characteristics of CCMs in biological samples represent a challenge due to enormous polarity difference, structural diversity, high structural similarity, and poor ionisation efficiency in mass spectrometry.

Methods An innovative mass spectrometry probe, 5-(diisopropylamino)amyline (DIAA), was developed for mapping the profile of CCMs using derivatization-liquid chromatographymass spectrometry.

Results With this innovative methodology, the sensitivity was enhanced up to 25,000-fold. Moreover, the hydrophobicity of polar CCMs, amino acids, TCA cycle intermediates and shortchain fatty acids, and the hydrophilicity of low-polar CCMs, long-chain fatty acids and bile acids, were significantly increased, resulting in a remarkable separation efficiency for which 68 different CCMs can be simultaneously determined. The polarity-reversal was revealed to be induced by the different impacts of aliphatic chains and nitrogen atom in DIAAA, the latter existing as a cation in the acidic mobile phase. Furthermore, a novel isotope labelled probe, DIAA- $d_{14}$, was also synthesised and utilised to develop an isotope labelling method based on DIAA/DIAA- $d_{14}$ pair for quickly and accurately hunting for the potential biomarkers in colorectal cancer (CRC), which is the second leading cause of death in the world. Finally, 52 CCMs were detected in the sera of CRC patients and 28 CCMs related with amino acids metabolism, TCA cycle, fatty acid metabolism, and gut flora metabolism were found to have a significant difference with that in healthy controls. The decrease of five SCFAs, including propionic acid (PA), butyric acid (BA), isobutyric acid (IBA), isovaleric acid (IVA) and valeric acid (VA) in the sera (figure 1). It was further confirmed in line with that of $\mathrm{Apc}^{\mathrm{Min} /+}$ mice, a wellestablished animal model of intestinal cancer.

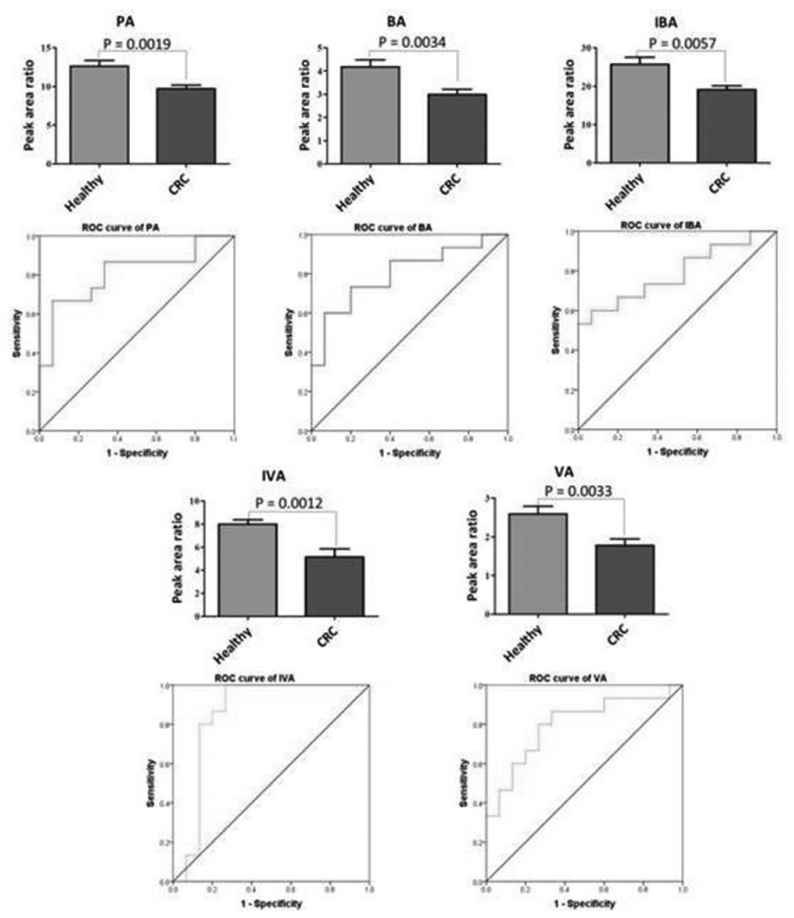

Abstract IDDF2018-ABS-0121 Figure 1

Conclusions These results clearly indicated a close relationship of the change of serum SCFAs with CRC for the first time. 